12,03

\title{
Сравнительное рентгеноабсорбционное исследование спектра свободных электронных состояний в комплексах тетрафенилпорфиринов кобальта и никеля
}

\author{
(С) Г.И. Свирский ${ }^{1}$, А.В. Генералов ${ }^{1,2}$, А.Ю. Клюшин ${ }^{1,3,4}$, К.А. Симонов ${ }^{1,2,5}$, С.А. Красников ${ }^{1,6}$, \\ Н.А. Виноградов ${ }^{1,2}$, А.Л. Тригуб ${ }^{7}$, Я.В. Зубавичус ${ }^{7}$, А.Б. Преображенский1,2, А.С. Виноградов ${ }^{1, \text { ฯ }}$
}

${ }^{1}$ Санкт-Петербургский государственный университет,

Санкт-Петербург, Россия

${ }^{2}$ MAX IV Laboratory, University of Lund, PO Box 118 ,

Lund, Sweden

${ }^{3}$ Research Group Catalysis for Energy, Helmholtz Zentrum Berlin,

Berlin, Germany

${ }^{4}$ Fritz-Haber-Institut der Max-Planck-Gesellschaft, Dept. Inorganic Chemistry,

Berlin, Germany

${ }^{5}$ Department of Physics and Astronomy, Uppsala University, Box 516,

Uppsala, Sweden

${ }^{6}$ School of Physical Sciences, Dublin City University, Glasnevin,

Dublin 9, Republic of Ireland

${ }^{7}$ Национальный исследовательский центр „Курчатовский институт“,

Москва, Россия

『 E-mail: asvinograd@gmail.com

(Поступила в Редакцию 3 июля 2017 г.)

Методами рентгеновской абсорбционной спектроскопии изучены энергетические распределения и свойства нижних свободных электронных состояний в комплексах тетрафенилпорфиринов кобальта СоТРР и никеля NiTPP. Квазимолекулярный анализ экспериментальных спектров поглощения, измеренных в области $2 p$ - и $1 s$-порогов ионизации комплексообразующих атомов металлов, а также $1 s$-порогов атомов лиганда (азота и углерода), выполнен на основе сравнения соответствующих спектров между собой и со спектрами простейшего порфирина никеля NiP. Установлено, что при общем подобии спектров азота и углерода для CoTPP и NiTPP тонкая структура $2 p$ - и $1 s$-спектров поглощения атомов кобальта и никеля отличается радикальным образом друг от друга. Наблюдаемые отличия спектров кобальта и никеля связываются с особенностями энергетического распределения свободных электронных состояний с $3 d$-характером. Наличие в СоТРР частично заполненной валентной $3 d b_{2 g}$-MO обусловливает появление в спектрах кобальта низкоэнергетической полосы, отсутствующей в спектрах никеля NiTPP, и приводит к дублетной структуре переходов на $b_{1 g}$ - и $e_{g}$-МО вследствие обменного взаимодействия между $3 d$-электронами в частично заполненных $3 d b_{2 g}$ - и $3 d b_{1 g}$ - (или $3 d e_{g}$-МО. Спектр свободных состояний СоТРР отличается от спектра NiTPP также за счет меньшего энергетического расстояния между $3 d b_{1 g}$-MO и $e_{g}$-МО и различного положения несвязывающих МО с С2p-характером порфинового лиганда.

Работа выполнена частично в рамках двухсторонней программы „Российско-Германская лаборатория БЭССИ““ при поддержке Российского фонда фундаментальных исследований (грант № 15-02-06369).

DOI: $10.21883 /$ FTT.2018.03.45564.217

\section{1. Введение}

Порфирины атомов переходных металлов вызывают большой научный и технологический интерес ввиду их ключевой роли в ряде биохимических процессов $[1,2]$ и возможностей использования в таких современных технологиях как молекулярная электроника [3], нелинейная оптика [4], катализ [5], диагностика и терапия раковых заболеваний [6], химические сенсоры [7], светособирающие комплексы [8] и устройства хранения информации [9]. В связи с этим атомная и электронная структура молекулярных комплексов металлопорфиринов является предметом большого количе- ства экспериментальных и теоретических исследований. Однако вследствие многообразия порфиринов и сложности их электронного строения эти исследования до настоящего времени охватывают в основном лишь узкий круг наиболее простых систем. К числу наиболее популярных объектов исследования относятся молекулярные комплексы тетрафенилпорфиринов переходных $3 d$-металлов (3d-МТРР).

Детали атомной и электронной структуры молекул $3 d$-МТРР исследовались с помощью методов сканирующей туннельной и электронной микроскопии, оптического поглощения в ИК, видимом и УФ диапазонах, а также фотоэлектронной спектроскопии. Большинство из 
этих работ были посвящены изучению морфологии и ориентации тонких пленок этих металлопорфиринов на различных монокристаллических поверхностях [10-14], влияния на электронную структуру молекул MTPPs их взаимодействия с различными металлическими подложками [10,15-17] или синтезу молекул $3 d$-МТРР из молекул свободного тетрафенилпорфирина $\mathrm{H}_{2} \mathrm{TPP}$ с помощью поверхностных реакций замещения центральных атомов водорода атомом металла подложки [18-20]. В то же время лишь в небольшом количестве работ исследовались энергетическое распределение и свойства свободных (занятых) электронных состояний $3 d$-МТРР, которые определяют все физико-химические свойства этих молекулярных комплексов.

Одним из наиболее информативных экспериментальных методов получения информации о свойствах свободных электронных состояний в различных полиатомных системах является в настоящее время метод рентгеновской абсорбционной спектроскопии, основанный на анализе ближней тонкой структуры рентгеновских спектров поглощения (БТС РСП или NEXAFS — Near Edge X-ray Absorption Fine Structure) [21]. В работах [22-26] NEXAFS спектроскопия была использована для изучения свободных электронных состояний в некоторых $3 d$-МТРР. Однако в них, как правило, рассматривались только либо $1 s$-спектры поглощения атомов порфиринового лиганда (азота и углерода), либо $1 s$ - или $2 p$-спектры поглощения центрального атома металла в $3 d$-МТРР. Наиболее полные рентгеноабсорбционные исследования были выполнены, по-видимому, для ряда порфиринов никеля в работах [27-29], продемонстрировавших возможности рентгеновской абсорбционной спектроскопии для характеризации атомного строения и электронных свойств подобных систем, а также особенностей химического связывания атомов в них.

Следует заметить, что рентгеновские спектры атомов металла и лиганда исследованных порфиринов никеля позволяют достаточно просто установить однозначную связь абсорбционных полос с электронными переходами на свободные молекулярные орбитали (МО) молекулярных комплексов, поскольку все их валентные МО полностью заполнены и соответственно полный спин $S$ молекул равен нулю. В случае порфиринов других $3 d$-атомов (Co, Fe и др.), имеющих среди валентных MO частично заполненные орбитали, молекулы обладают парамагнитными свойствами, поскольку их полный спин отличен от нуля. В этом случае спектры атомов металлов будут существенно усложняться вследствие обменного $3 d-3 d$-электронного взаимодействия между внутренним электроном, перешедшим на свободную МО, и электронами в частично заполненной валентной MO. Paнее это было продемонстрировано на примере NEXAFS спектров фталоцианинов $3 d$-атомов [30]. Как следствие, эти обменные эффекты заметно затрудняют полную идентификацию спектров и получение информации о свободных электронных состояниях изучаемой полиатомной системы.
Основной целью данной работы являлось получение для молекулярных комплексов тетрафенилпорфиринов кобальта и никеля, СоTPP и NiTPP, детальной информации о свойствах низших свободных электронных состояний на основе сравнительного анализа полного набора рентгеновских спектров поглощения атомов металла $(\mathrm{Co} 2 p-$, $\mathrm{Co} 1 s-$-, Ni2p- и $\mathrm{Ni} 1 s-)$ и порфиринового лиганда $(\mathrm{N} 1 s-$ и $\mathrm{C} 1 s-)$ с учетом влияния различия спинового состояния молекул СоТРP $(S=1 / 2)$ и NiTPP $(S=0)$ на тонкую структуру $2 p$ - и $1 s$-спектров атомов кобальта. При анализе предполагается использование результатов проведенного ранее рентгеноабсорбционного исследования простейшего порфирина никеля $\mathrm{NiP}$ [29].

\section{2. Экспериментальная часть}

Измерения $\mathrm{Ni} 2 p-, \mathrm{Co} 2 p$-, $\mathrm{N} 1 s$ - и $\mathrm{C} 1 s$-спектров поглощения NiTPP и CoTPP были выполнены с использованием оборудования Российско-Германского канала вывода и монохроматизации синхротронного излучения (СИ) электронного накопителя БЭССИ II (BESSY II, Berlin, Germany) [31]. Порошки исследованных порфиринов были приобретены в Sigma Aldrich [32]. Образцы для измерений были приготовлены in situ путем термического испарения порошков порфиринов из танталового тигля ячейки Кнудсена и осаждения на чистую поверхность медной пластинки (NiTPP) или грань (111) кристалла иридия (СоТРР). Приготовленные образцы представляли собой поликристаллические слои с толщинами около $30 \mathrm{~nm}$ (NiTPP) и $5 \mathrm{~nm}$ (CoTPP). Температура тигля при напылении была около $600 \mathrm{~K}$. Скорость напыления контролировалась кварцевыми микровесами и составляла около 0.6 и $0.1 \mathrm{~nm} / \mathrm{min}$ для слоев NiTPP и CоTPP соответственно. Давление паров порфиринов в препарационной камере при напылении не превышало $5 \cdot 10^{-8}$ mbar.

Рентгеновские Ni2p-, Co2p-, N1s- и C $1 s$-спектры были получены методом полного электронного выхода внешнего рентгеновского фотоэффекта в режиме измерения тока утечки с образца при варьировании энергии фотонов, падающих на образец. Известно, что значение полного электронного выхода вблизи порогов ионизации пропорционально сечению поглощения [33,34]. Энергетическое разрешение монохроматора в области $\mathrm{Ni} 2 p_{3 / 2^{-}}$ $(\sim 850 \mathrm{eV}), \mathrm{Co} 2 p_{3 / 2^{-}}(\sim 780 \mathrm{eV}), \mathrm{N} 1 s-(\sim 400 \mathrm{eV})$ и $\mathrm{C} 1 s-$ $(\sim 280 \mathrm{eV})$ краев поглощения составляло 550, 480, 180 и $100 \mathrm{meV}$ соответственно. Все спектры нормировались на падающий поток фотонов, который регистрировался путем измерения фототока с золотой сетки, установленной на выходе канала вывода СИ. Энергия фотонов калибровалась с помощью положений первых узких пиков в Ti2 $p$ - $(459.0 \mathrm{eV})$ и $\mathrm{F} 1 s-(683.9 \mathrm{eV})$ спектрах поглощения твердофазного $\mathrm{K}_{2} \mathrm{TiF}_{6}$ [35]. Измерения проводились при комнатной температуре и давлении остаточных газов в экспериментальной камере не выше $2 \cdot 10^{-10} \mathrm{mbar}$. В процессе измерений заметных эффектов зарядки и разложения образцов, облучаемых интенсивным пучком 
монохроматизированного СИ мягкого рентгеновского диапазона, не наблюдалось.

Рентгеновские M1s-спектры поглощения NiTPP и СоТРР были измерены методом пропускания, используя оборудование станции „Структурное материаловедение“ канала вывода и монохроматизации $1.3 \mathrm{~b}$ накопительного кольца „Сибирь-2“ Курчатовского специализированного источника СИ (КИСИ-Курчатов) [36]. В качестве детекторов падающего и проходящего рентгеновского излучения использовались ионизационные камеры, заполненные смесью газов $\left(\mathrm{N}_{2}, \mathrm{Ar}, \mathrm{Xe}\right)$. Образцы для измерений представляли собой однородные порошки порфиринов, засыпанные в кюветы толщинами $\sim 0.5 \mathrm{~mm}$, оба торца которых были ограничены каптоновой пленкой. Энергетическое разрешение монохроматора в области Ni1s$(\sim 8350 \mathrm{eV})$ и $\mathrm{Co} 1 s-(\sim 7700 \mathrm{eV})$ краев поглощения составляло 900 и $800 \mathrm{meV}$ соответственно [36].

\section{3. Результаты и обсуждение}

Исследованные тетрафенилпорфирины атомов $3 d$-металла $\mathrm{C}_{44} \mathrm{H}_{28} \mathrm{~N}_{4} \mathrm{M}(\mathrm{M}=\mathrm{Ni}, \mathrm{Co})$ представляют собой молекулярные комплексы, состоящие из плоской молекулы порфина металла МР, к которой в мезо-позициях к мостиковым атомам углерода $\mathrm{C}_{b}$ присоединены четыре фенильных группы $\mathrm{C}_{6} \mathrm{H}_{5}$ (рис. 1) [37]. В свою очередь порфин металла образован центральным комплексообразующим атомом металла М, который окружен четырьмя пиррольными кольцами $\mathrm{NC}_{4}$, соединенными между собой атомами $\mathrm{C}_{\alpha}$ через мостиковые атомы углерода $\mathrm{C}_{b}$. Плоские фенильные группы в молекуле МТРР, замещающие в МР атомы водорода у атомов $\mathrm{C}_{b}$, ориентированы перпендикулярно относительно плоскости порфинового макроцикла вследствие межэлектронного отталкивания между атомами водорода макроцикла и фенильных групп [38]. В конденсированном состоянии комплексы представляют собой молекулярные кристаллы, в которых молекулы сцеплены друг с другом за счет $\pi-\pi$ взаимодействия делокализованных $\pi$-орбиталей порфинового макроцикла, определяемого главным образом силами Ван-дер-Ваальса [39]. В кристаллах угол поворота фенильных групп отличается от $90^{\circ}$ и точечная симметрия MTPPs в зависимости от угла поворота характеризуется группами $\mathrm{S}_{4}$ или $\mathrm{D}_{2 d}$ [37-39]. Тем не менее, центральная часть комплекса, включающая атом металла и порфиновый макроцикл, сохраняет плоскую конфигурацию, соответствующую группе $\mathrm{D}_{4 h}$ точечной симметрии.

Переходя к рассмотрению экспериментальных спектров поглощения изучаемых комплексов, заметим, что CoTPP и NiTPP имеют близкие параметры атомного строения, а их электронные конфигурации отличаются всего одним $3 d$-электроном, отсутствующим в конфигурации атома кобальта $\left([\operatorname{Ar}] 3 d^{7} 4 s^{2}\right)$ по сравнению с атомом никеля $\left([\mathrm{Ar}] 3 d^{8} 4 s^{2}\right)$. Последнее указывает на важность изучения спектра М3d-электронных состояний в этих полиатомных системах. Наиболее эффективным

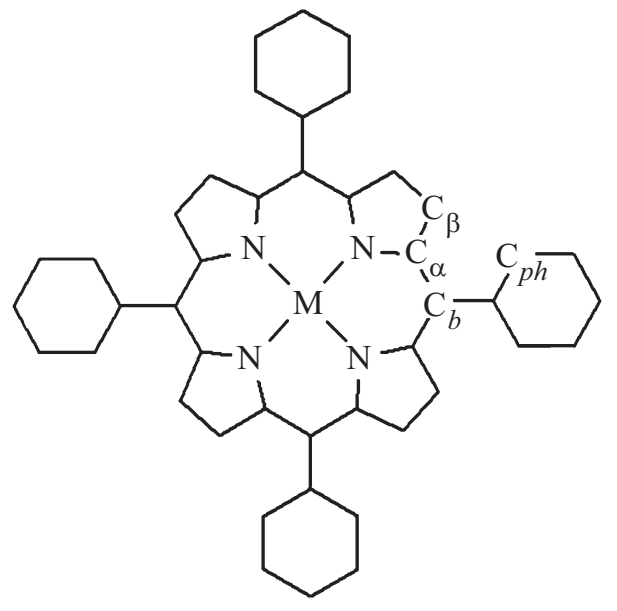

Рис. 1. Схематическое изображение комплекса тетрафенилпорфирина металла МТРP $(\mathrm{M}=\mathrm{Ni}, \mathrm{Co})$.

способом получения этой информации для свободных $3 d$-состояний является рентгеновская абсорбционная спектроскопия в области $2 p$-краев поглощения атомов $3 d$-металлов. Тонкая структура этих спектров формируется в основном дипольно-разрешенными переходами $2 p_{3 / 2^{-}}$и $2 p_{1 / 2}$-электронов в незанятые состояния, образованные с участием $3 d$-состояний атомов металла, поскольку разрешенные переходы $2 p$-электронов в $4 s$-состояния примерно в 20 раз менее интенсивные по сравнению с $2 p \rightarrow 3 d$-переходами [40].

Поэтому обсуждение полученных экспериментальных результатов начнем со сравнительного анализа M2p-спектров поглощения комплексов NiTPP и CoTPP (рис. 2, левая панель). Обозначения абсорбционных полос ближней тонкой структуры в этих (и других) спектрах комплексов выполнены с учетом результатов их последующей идентификации. Оба спектра состоят из двух групп абсорбционных полос, отражающих дипольноразрешенные переходы $\mathrm{M} 2 p_{3 / 2^{-}}$и $2 p_{1 / 2}$-электронов в свободные электронные состояния, образованные с участием $3 d$-состояний атомов никеля (кобальта). Заметим, что $2 p$-спектры поглощения никеля в NiTPP практически совпадают со спектрами порфирина NiP [29]. В дальнейшем мы будем рассматривать только низкоэнергетические $2 p_{3 / 2}$-компоненты, которые имеют более четкую и разрешенную тонкую структуру. В $2 p_{1 / 2}$-спектре последняя разрешена существенно хуже вследствие значительно бо́льшей естественной ширины $\mathrm{M} 2 p_{1 / 2}$-уровня, что обусловлено дополнительными Оже-процессами распада рентгеновского (дырочного) $2 p_{1 / 2}$-состояния атома металла $\left(2 p_{1 / 2}^{-1} \rightarrow 2 p_{3 / 2}^{-1} 3 d^{-1}\right)$ [41].

При сравнении M $2 p_{3 / 2}$-спектров поглощения NiTPP и СоТРР хорошо видно, что их тонкая структура сильно отличается друг от друга. Спектр никеля характеризуется интенсивным низкоэнергетическим пиком $A$ и малоинтенсивными полосами $B-D$ при бо́льших энергиях фотонов. В случае же Co2 $p_{3 / 2}$-спектра в низкоэнергети- 

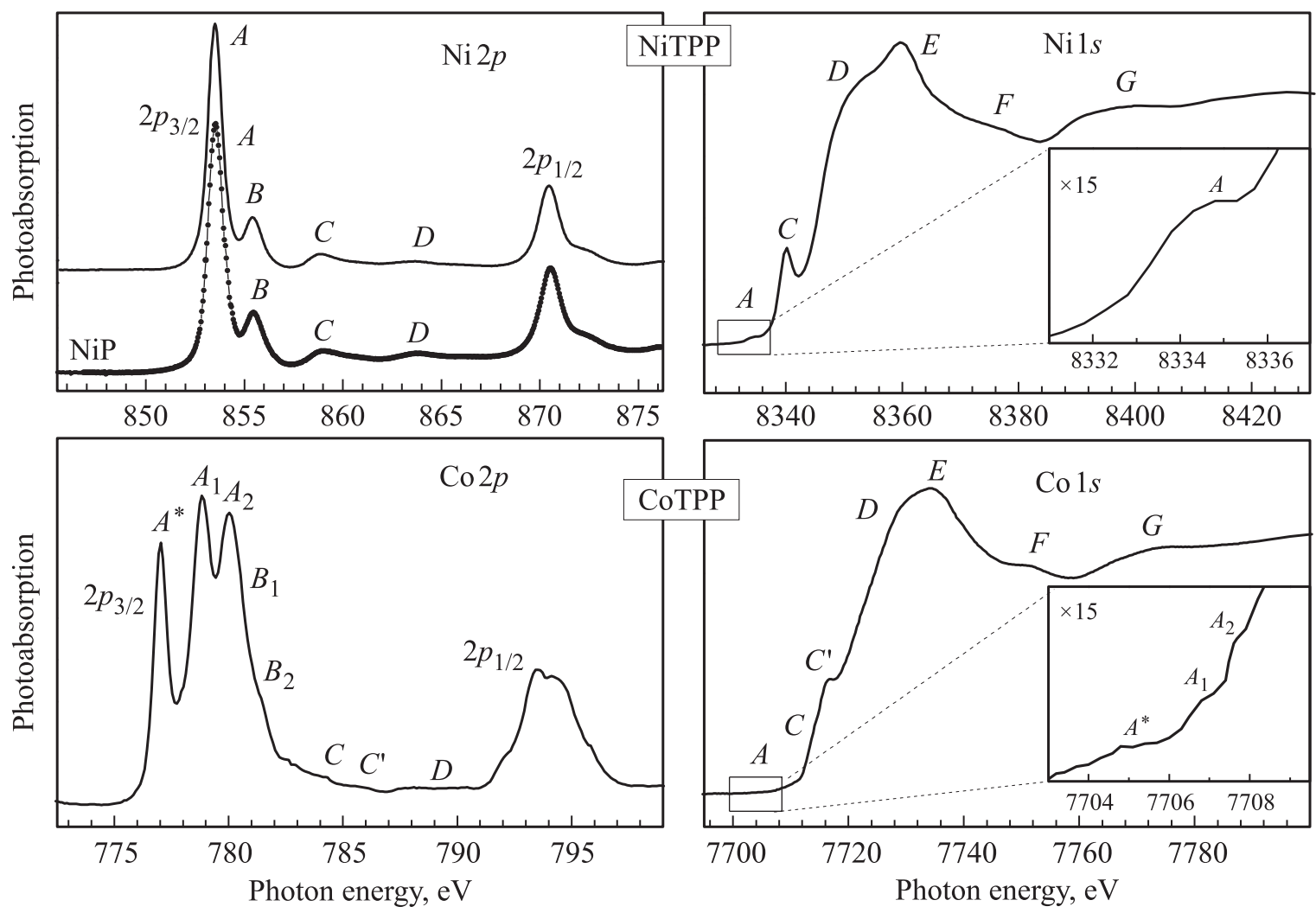

Рис. 2. М2p- и M1s-спектры поглощения тетрафенилпорфиринов никеля и кобальта NiTPР и СоТРР. Низкоэнергетическая область $\mathrm{M} 1 s$-спектров в увеличенном масштабе изображена на вставках. Для сравнения приведен Ni2p-спектр порфирина NiP [29].

ческой области наблюдаются три интенсивных близко расположенных резонанса $A^{*}, A_{1}$ и $A_{2}$. Далее, с высокоэнергетической стороны пика $A_{2}$ видны два плеча $B_{1}$ и $B_{2}$, после которых следуют полосы поглощения $C-C^{\prime}$ и $D$. Поскольку сравниваемые спектры доминируются переходами остовных М2p $3 / 2$-электронов в свободные электронные состояния с вкладами М3d-состояний, то очевидно, что различия $2 p_{3 / 2}$-спектров никеля и кобальта, наблюдаемые в их низкоэнергетической области, обусловлены особенностями энергетического распределения в NiTPP и СоТРP свободных электронных состояний с М3 $d$-вкладами.

Это соображение согласуется с результатами рассмотрения для изучаемых комплексов $1 s$-спектров поглощения атомов никеля и кобальта (рис. 2, правая панель), тонкая структура которых главным образом формируется дипольно-разрешенными переходами $\mathrm{M} 1 s$-электронов в свободные электронные состояния, имеющие вклады дискретных $4 p$ - и континуумных $\varepsilon p$-состояний атомов металла. Действительно, хорошо видно, что Ni1sи $\mathrm{Co} 1 s$-спектры комплексов демонстрируют подобное спектральное поведение, а небольшие различия между ними в основном сводятся к меньшей контрастности абсорбционных структур в Co1s-спектре, двойной полосе $C\left(C\right.$ и $\left.C^{\prime}\right)$ и различиям в структуре слабых квадрупольных $\mathrm{M} 1 s \rightarrow 3 d$ переходов в низкоэнергетической области спектров (вставки на рисунках). Инте- ресно заметить, что переход от Ni1s- к Co1s-спектру сопровождается, как и в случае М2p-спектров, усложнением низкоэнергетической структуры и появлением трех абсорбционных полос $A^{*}, A_{1}$ и $A_{2}$ вместо одной $A$, обусловленных квадрупольными $1 s \rightarrow 3 d$-переходами.

Сравним теперь $1 s$-спектры поглощения атомов лиганда (азота и углерода) в комплексах NiTPP и СоTPP (рис. 3). Предварительно заметим, что измеренные спектры по числу абсорбционных полос и их энергетическим положениям хорошо согласуются с $\mathrm{N} 1 s-$ и $\mathrm{C} 1 s$-спектрами тетрафенилпорфиринов ZnTPP и СоTPР, полученными в работе [26]. На рисунке для сравнения приведены также соответствующие спектры порфирина $\mathrm{NiP}$ [29]. На левой панели хорошо видно, что тонкая структура $\mathrm{N} 1 s$-спектров поглощения практически совпадает для комплексов никеля: при переходе от простейшего порфирина $\mathrm{NiP}$ к более сложному NiTPP основная полоса поглощения $B$ сохраняет свою форму и энергетическое положение в спектре $(398.73 \mathrm{eV})$, следующая полоса $C^{*}$ выравнивает свою интенсивность относительно $C$, и для всех полос $C^{*}-D$ наблюдаются незначительные (в пределах $0.15 \mathrm{eV}$ ) энергетические сдвиги. В случае СоТРP спектральные характеристики тонкой структуры $\mathrm{N} 1 s$-спектра также изменяются достаточно слабо при сохранении общего спектрального хода поглощения. Так, резонанс $B$ демонстрирует высокоэнергетический сдвиг $\sim 0.25 \mathrm{eV}$, полоса $C^{*}$ становится более интенсив- 




Pис. 3. $\mathrm{N} 1 s-$ и $\mathrm{C} 1 s$-спектры поглощения тетрафенилпорфиринов никеля и кобальта NiTPP и СоТРP. Для сравнения приведены $\mathrm{N} 1 s$ - и $\mathrm{C} 1 s$-спектры порфирина $\mathrm{NiP}[29]$. Вертикальными отрезками показаны основные переходы в С1s-спектре поглощения молекулы бифенила [42].

ной нежели $C$ и смещается на $0.3 \mathrm{eV}$ в сторону бо́льших энергий, для остальных полос поглощения $C-D$ также характерны небольшие высокоэнергетические сдвиги.

При сравнении $\mathrm{C} 1 s$-спектров поглощения (рис. 3, правая панель) видно, что тонкая структура спектра радикальным образом перестраивается при переходе от NiP к NiTPP. B результате в спектре последнего сохраняются, по всей видимости, лишь полосы $B$ и $D$ спектра NiP. Полосы $B_{1}, B_{2}, C^{*}$ и $C$ спектра NiP заплывают (или совсем исчезают), и появляется целый ряд новых абсорбционных полос $B_{1}^{*}, B_{2}^{*}, B^{\prime}, C_{1}^{*}$ и $C_{2}^{*}$. Наблюдаемые изменения в тонкой структуре $\mathrm{C} 1 s$-спектра NiTPP, с одной стороны, отражают изменения в спектре свободных состояний порфина никеля, обусловленные его химическим связыванием с четырьмя фенильными группами $\mathrm{C}_{6} \mathrm{H}_{5}$, а с другой - появление в спектре $\mathrm{C} 1 s$-возбуждений фенильных групп. Непосредственное сравнение спектра NiTPP с $\mathrm{C} 1 s$-спектром молекулы бифенила $\left(\mathrm{C}_{6} \mathrm{H}_{5}\right)_{2}$ [42], основные полосы поглощения которой показаны вертикальными черточками на шкале энергий (рис. 3), указывает на связь абсорбционных резонансов $B^{\prime}, C_{1}^{*}, C_{2}^{*}$ и $D$ со свободными $1 \pi^{*}-, \sigma^{*}(\mathrm{C}-\mathrm{H})$-, $2 \pi^{*}$ - и $\sigma^{*}(\mathrm{C}-\mathrm{C})$-состояниями фенильной группы. Средние межатомные расстояния в пиррольном и фенильном (бензольном) циклах примерно одинаковы $(\sim 0.139 \mathrm{~nm})$, поэтому $\sigma^{*}(\mathrm{C}-\mathrm{C})$-состояния в $\mathrm{C} 1 s$-спектрах поглощения имеют близкие энергетические положения. Низкоэнергетическая полоса $B_{1}^{*}-B_{2}^{*}$, наблюдаемая только в $\mathrm{C} 1 s$-спектре, связана, по всей видимости, с электронными переходами на несвязывающую МО порфинового лиганда с C2p-характером. Эта идентификация полосы $B_{1}^{*}-B_{2}^{*}$ согласуется с результатами расчета $\mathrm{C} 1 s$-спектра ZnTPP и CoTPP методом функционала плотности (DFT) [26].

Переходя к рассмотрению $\mathrm{C} 1 s$-спектра поглощения CоTPР, замечаем, что тонкая структура этого спектра по спектральному поведению и энергетическим положениям абсорбционных полос совпадает со структурой спектра NiTPP.

Выполненное непосредственное сравнение всех рентгеновских спектров поглощения комплексов кобальта и никеля указывает на определяющую роль порфина металла в формировании тонкой структуры спектров поглощения атомов $\mathrm{Ni}, \mathrm{Co}$ и $\mathrm{N}$, тогда как структура $\mathrm{C} 1 s$-спектров NiTPP и СоТРP определяется главным образом рентгеновскими возбуждениями атомов углерода фенильных групп, а возбуждения порфинового макроцикла наблюдаются явно лишь в низкоэнергетической части спектров. С этим предположением также вполне согласуется обнаруженное слабое влияние на 
спектры атомов лиганда замены комплексообразующего атома металла.

С учетом этих наблюдений представляется логичным выполнить идентификацию полос поглощения в разных спектрах изучаемых комплексов в рамках квазимолекулярного подхода [35], согласно которому нижние электронные состояния полиатомной системы могут быть приближенно описаны свободными молекулярными орбиталями (MO) квазимолекулы, образуемой поглощающим атомом и атомами его ближайшего окружения. Результаты этой идентификации используем затем для характеризации нижних незанятых электронных состояний в СоТРP и NiTPP.

Химическое связывание центрального комплексообразующего атома металла с атомами азота порфинового лиганда в NiP, NiTPP и CoTPP имеет донорноакцепторный характер и осуществляется за счет смещения неподеленной $2 p$-электронной пары каждого из атомов азота на свободные $3 d$-состояния атома металла $[38,43]$. В результате образуются четыpe $s p^{2} d$-гибридизированные $\sigma$-связи, обеспечивающие плоскую квадратную $\left(\mathrm{D}_{4 h}\right)$ координацию атома металла атомами азота в исследованных комплексах. Как было показано ранее [29], атом металла и ближайшие к нему атомы азота и углерода $\mathrm{C}_{\alpha}$ в порфирине $\mathrm{NiP}$ можно рассматривать как квазимолекулу $\mathrm{MN}_{4}\left(\mathrm{C}_{\alpha}\right)_{8}$, которая имеет точечную симметрию комплекса $\left(\mathrm{D}_{4 h}\right)$ и определяет основные абсорбционные резонансы в спектрах поглощения NiP. Вследствие совпадения спектров атомов никеля и азота для NiP и NiTPP логично использовать эту квазимолекулу и для интерпретации спектров атомов металла и азота в комплексах МТРР. В рамках этого подхода абсорбционные полосы во всех спектрах атомов $\mathrm{Ni}, \mathrm{Co}, \mathrm{N}$ и $\mathrm{C}_{\alpha}$ в NiTPP и CoTPP могут быть приближенно описаны с помощью переходов остовных $1 s$ - и $2 p$-электронов этих атомов на свободные $\mathrm{MO}$ квазимолекулы $\mathrm{MN}_{4}\left(\mathrm{C}_{\alpha}\right)_{8}$, которые образуются за счет ковалентного смешивания между валентными $\mathrm{M} 3 d, 4 s, 4 p$-, $\mathrm{N} 2 p$ - и $\mathrm{C}_{\alpha} 2 p$-атомными орбиталями $(\mathrm{AO})$. Для полного описания структуры $\mathrm{C} 1 s$-спектров NiTPP и CoTPP необходимо учесть также рентгеновские $1 s$-возбуждения остальных атомов углерода - восьми пиррольных $\mathrm{C}_{\beta}$, четырех мостиковых $\mathrm{C}_{b}$ и двадцати четырех фенильных $\mathrm{C}_{p h}$. Последние будут давать доминирующий вклад в тонкую структуру $\mathrm{C} 1 s$-спектра поглощения изучаемых комплексов.

Обсудим сначала природу $2 p$-возбуждений атомов никеля и кобальта, которые отражают распределение незанятых $3 d$-электронных состояний в NiTPP и CoTPP (рис. 2, левая панель). В кристаллическом поле с симметрией плоского квадрата $\left(\mathrm{D}_{4 h}\right)$, образуемого четырьмя атомами азота, пять изначально вырожденных М3d-AО расщепляются на двукратно вырожденную $e_{g}\left(3 d_{x z, y z}\right)$ - и невырожденные $a_{1 g}\left(3 d_{z^{2}}\right)$-, $b_{2 g}\left(3 d_{x y}\right)$ и $b_{1 g}\left(3 d_{x^{2}-y^{2}}\right)$-компоненты [44]. Таким образом, двухвалентные атомы никеля $\mathrm{Ni}$ (II) и кобальта $\mathrm{Co}(\mathrm{II})$ в NiTPP и СоТРР имеют электронные конфигурации $\mathrm{Ni}^{2+}$ и $\mathrm{Co}^{2+}$ ионов, $\left(e_{g}\right)^{4}\left(a_{1 g}\right)^{2}\left(b_{2 g}\right)^{2}\left(b_{1 g}\right)^{0}$ и $\left(e_{g}\right)^{4}\left(a_{1 g}\right)^{2}\left(b_{2 g}\right)^{1}\left(b_{1 g}\right)^{0}$ соответственно. Как следствие, атом никеля в NiTPP имеет полностью заполненные три АО и характеризуется суммарным спином, равным нулю $(S=0)$, в то время как в СоТРР имеется один неспаренный электрон на $3 d b_{2 g}$-орбитали, и комплекс является парамагнетиком $(S=1 / 2)$.

Эти $3 d$-АО никеля и кобальта, в свою очередь, смешиваются с N2p-AO, формируя МО $\sigma$-типа $-a_{1 g}$ и $b_{1 g}-$ и $\pi$-типа $-b_{2 g}$ и $e_{g}$. Если учесть, что М4p-AО также принимают участие в связывании, то это приводит к образованию $\sigma e_{u^{-}}, \pi a_{2 u^{-}}$и $\pi e_{u^{-}}$МО. Стоит отметить также еще две МО $\pi$-типа, $a_{2 g}$ и $b_{2 u}$, которые образуются из $\mathrm{N} 2 p$-АО и являются несвязывающими [43].

Таким образом, в комплексе NiTPP имеется только одна полностью свободная $3 d b_{1 g}-\mathrm{MO}$, а в СоТРР кроме нее присутствует еще частично заполненная $3 d b_{2 g}$-MO. В результате в $2 p_{3 / 2}$-спектре поглощения никеля наблюдается всего один дипольно-разрешенный $2 p_{3 / 2} \rightarrow 3 d b_{1 g}$ переход, который отвечает за основной резонанс $A$ в спектре. В рамках данного подхода в $\mathrm{Co} 2 p_{3 / 2}$-спектре ожидаются два абсорбционных резонанса $A^{*}$ и $A$, которые связаны с переходами $2 p_{3 / 2}$-электронов в частично заполненное $3 d b_{2 g^{-}}$и незаполненное $3 d b_{1 g}$-состояния иона кобальта. Однако в конечном состоянии второго перехода $\left(3 d b_{2 g}^{1} 3 d b_{1 g}^{1}\right)$ между $3 d$-электронами в частично заполненных $3 d b_{2 g}$ - и $3 d b_{1 g}$-МО возникает дополнительное (обменное) взаимодействие, которое расщепляет конечное состояние на два - синглетное $(S=0)$ и триплетное $(S=1)$, приводя к появлению в спектре двух резонансов $A_{1}$ и $A_{2}$ вместо одного $A$. Согласно правилу Хунда [43], наименьшей энергией обладает терм с наибольшим спином: обменное взаимодействие между электронами с параллельными спинами уменьшает энергию состояния. Таким образом, пик $A_{1}$ соответствует переходу в триплетное возбужденное состояние с суммарным спином, равным единице $\left(b_{2 g}^{1} \uparrow b_{1 g}^{1} \uparrow, S=1\right)$, а пик $A_{2}$ - переходу в синглетное возбужденное состояние $\left(b_{2 g}^{1} \uparrow b_{1 g}^{1} \downarrow, S=0\right)$. Следует подчеркнуть, что аналогичное усложнение тонкой структуры наблюдается и в M1s-спектрах при переходе от NiTPP к CoTPP (рис. 2, вставки на правой панели) в низкоэнергетической области, которая отражает квадрупольные $\mathrm{M} 1 s \rightarrow 3 d$ переходы. В свете вышеизложенного понятно, что изменение структуры квадрупольных переходов в СоТРР есть результат обменного $3 d-3 d$ электронного взаимодействия в конечном состоянии абсорбционного $\mathrm{Co} 1 s \rightarrow 3 d b_{1 g}$ перехода.

Обменное $3 d-3 d$-электронное взаимодействие приводит к расщеплению не только рентгеновских возбуждений, связанных с переходами $1 s-$ и $2 p_{3 / 2}$-электронов кобальта на $3 d b_{1 g}$-MO, но и усложняет абсорбционный переход $B$. Видно (рис. 2, левая панель), что одиночный резонанс $B$ в $2 p_{3 / 2}$-спектре NiTPP превращается в абсорбционные структуры (плечи) $B_{1}$ и $B_{2}$ с высокоэнергетической стороны резонанса $A_{2}$ в спектре 
поглощения CoTPP. В спектре NiTPP полоса $B$ отражает переходы $\mathrm{Ni} 2 p_{3 / 2}$-электронов на $e_{g}$-MO $\pi$-типа с вкладами $\mathrm{Ni} 3 d_{x z, y z} \pi$-состояний и $2 p \pi$-состояний атомов порфинового макроцикла. Это непосредственно следует из совпадения спектров никеля и азота для NiTPP и NiP и результатов анализа спектров последнего [29]. Учитывая $3 d$-характер незанятого $e_{g}$-состояния, естественно полагать, что $\mathrm{Co} 2 p_{3 / 2} \rightarrow 3 d e_{g}$ переход в СоТРР также будет дублетным вследствие формирования триплетного $\left(b_{2 g}^{1} \uparrow e_{g}^{1} \uparrow, S=1\right) B_{1}$ и синглетного $\left(b_{2 g}^{1} \uparrow e_{g}^{1} \downarrow, S=0\right)$ $B_{2}$ конечных состояний за счет обменного $3 d-3 d$ электронного взаимодействия. Квадрупольные $\mathrm{Co} 1 s \rightarrow 3 d e_{g}$ переходы в Cols-спектре незаметны вследствие очень малой интенсивности.

Заметим также, что мультиплетный характер конечных состояний для рентгеновских абсорбционных переходов, обусловленный обменным $3 d-3 d$ электронным взаимодействием, наблюдался ранее в $2 p_{3 / 2}$-спектрах атомов металла в подобных фталоцианиновых комплекcax $\mathrm{CoPc}$ и $\mathrm{FePc}$, которые обладают парамагнитными свойствами [30]. Известно, что мультиплетность рентгеновских возбуждений может быть обусловлена также обменным $2 p-2 p$ электронным взаимодействием, как это имеет место в случае O1s-спектра поглощения парамагнитной молекулы кислорода $\mathrm{O}_{2}[45,46]$.

Дальнейшее обсуждение тонкой структуры измеренных спектров продолжим, рассматривая в рамках квазимолекулярного подхода одновременно все спектры для NiTPP (рис. 4) и СоTPP (рис. 5). Для этого спектры представлены на энергетических шкалах, которые совмещены друг с другом по положению уровня Ферми с использованием энергий связи для остовных электронов. При таком совмещении спектров их тонкую структуру можно рассматривать как результат дипольноразрешенных переходов остовных электронов на общую систему незанятых электронных состояний, которые могут быть аппроксимированы свободными МО квазимолекулы, образуемой поглощающим атомом и атомами его ближайшего окружения [35].

$\mathrm{Ni} 2 p_{3 / 2^{-}}$, N1s- и $\mathrm{C} 1 s$-спектры NiTPP (рис. 4) представлены на своих энергетических шкалах, которые совмещены, используя разности энергий связи остовных уровней $\Delta E\left(\mathrm{Ni} 2 p_{3 / 2}-\mathrm{N} 1 s\right)=456.5 \mathrm{eV}$ и $\Delta E(\mathrm{~N} 1 s-\mathrm{C} 1 s)=113.8 \mathrm{eV}$, измеренных методом фотоэлектронной спектроскопии. Заметим, что полученные величины всего в пределах $0.1-0.3 \mathrm{eV}$ отличаются от данных работ для NiTPP $[11,48]$ и для $\mathrm{NiP}[29]$. Bo всех XPS измерениях для NiTPP фотоэлектронный $\mathrm{C} 1 s$-спектр представляет одиночную асимметричную с низкоэнергетической стороны линию, которая малопригодна для разложения на компоненты, связанные с атомами углерода в разных химических состояниях $\mathrm{C}_{\alpha}, \mathrm{C}_{\beta}, \mathrm{C}_{b}$ и $\mathrm{C}_{p h}$ (рис. 1) - и имеющие энергии связи в пределах ширины $(\sim 1 \mathrm{eV})$ экспериментальной линии. Таким образом, понятно, что использование $\mathrm{C} 1 s$-спектра при рассмотрении спектров атомов никеля
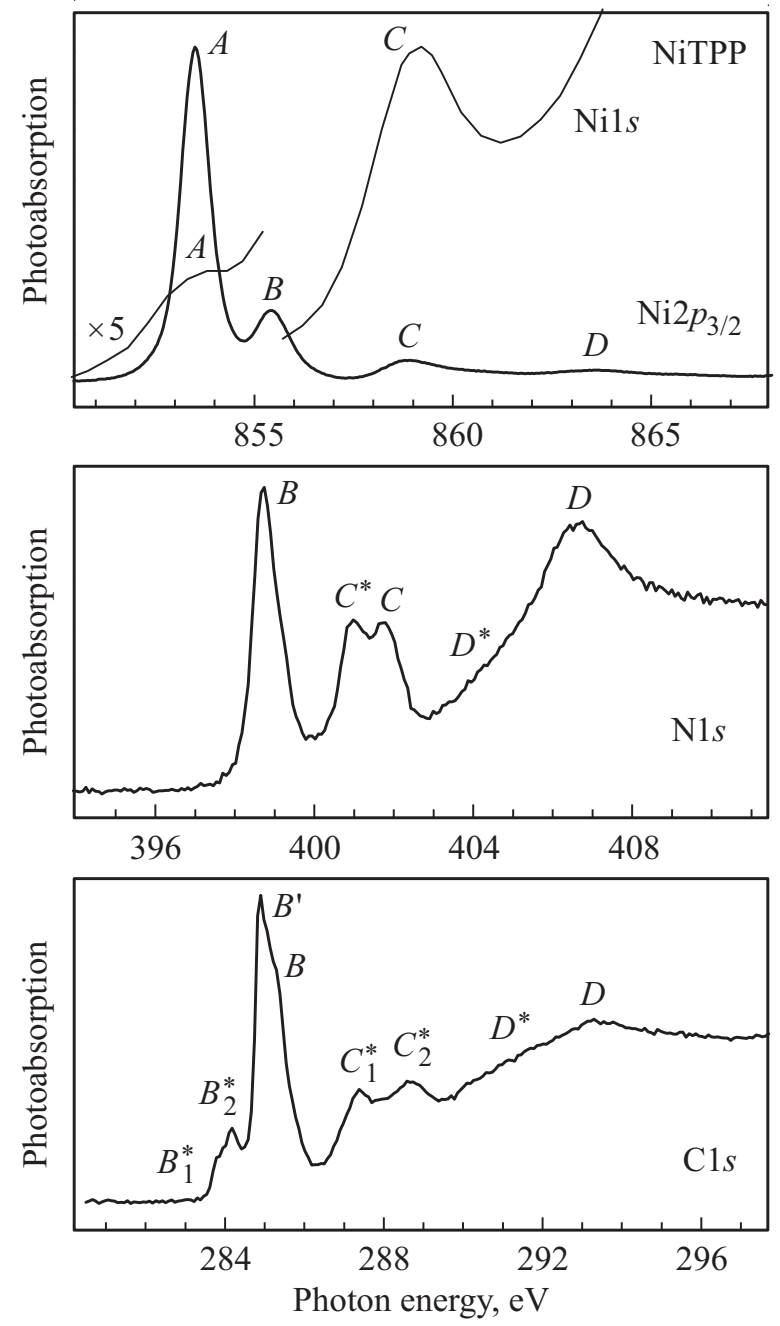

Рис. 4. Энергетически совмещенные $\mathrm{Ni} 2 p_{3 / 2^{-}}$, Ni1s-, $\mathrm{N} 1 s$ - и $\mathrm{C} 1 s$-спектры поглощения тетрафенилпорфирина никеля NiTPP. Энергетические шкалы совмещены с учетом разницы между энергиями связи остовных уровней $\Delta E\left(\mathrm{Ni} 2 p_{3 / 2}-\mathrm{N} 1 s\right)=456.5 \mathrm{eV}$ и $\Delta E(\mathrm{~N} 1 s-\mathrm{C} 1 s)=113.8 \mathrm{eV}$. $\mathrm{Ni} 1 s$-спектр приведен к шкале $\mathrm{Ni} 2 p_{3 / 2}$-спектра, используя энергию характеристической $K \alpha_{1}\left(1 s \rightarrow 2 p_{3 / 2}\right)$-перехода в металлическом никеле $7478.15 \mathrm{eV}$ [47]).

и азота NiTPP малоэффективно, поскольку его энергетическое совмещение выполнено по положению линии, которое, по всей видимости, отражает энергетическое положение фенильных атомов углерода, доминирующих в молекулярном комплексе. Энергетическое совмещение $\mathrm{Ni} 1 s$-спектра поглощения с $\mathrm{Ni} 2 p_{3 / 2}$-спектром выполнено, используя энергию рентгеновской характеристической $K \alpha_{1}\left(1 s \rightarrow 2 p_{3 / 2}\right)$-линии для металлического никеля $(7478.15 \mathrm{eV}[47])$, поскольку ее химсдвиг для подобных комплексов близок к нулю [49].

Рассмотрим все спектры NiTPP в рамках квазимолекулярного подхода, считая, что основные резонансы в них могут быть аппроксимированы свободными МО квазимолекулы $\mathrm{NiN}_{4}\left(\mathrm{C}_{\alpha}\right)_{8}$. Из рис. 4 отчетливо видно, что низкоэнергетическая полоса $A$ в Ni2 $2 p_{3 / 2}$-спектре погло- 

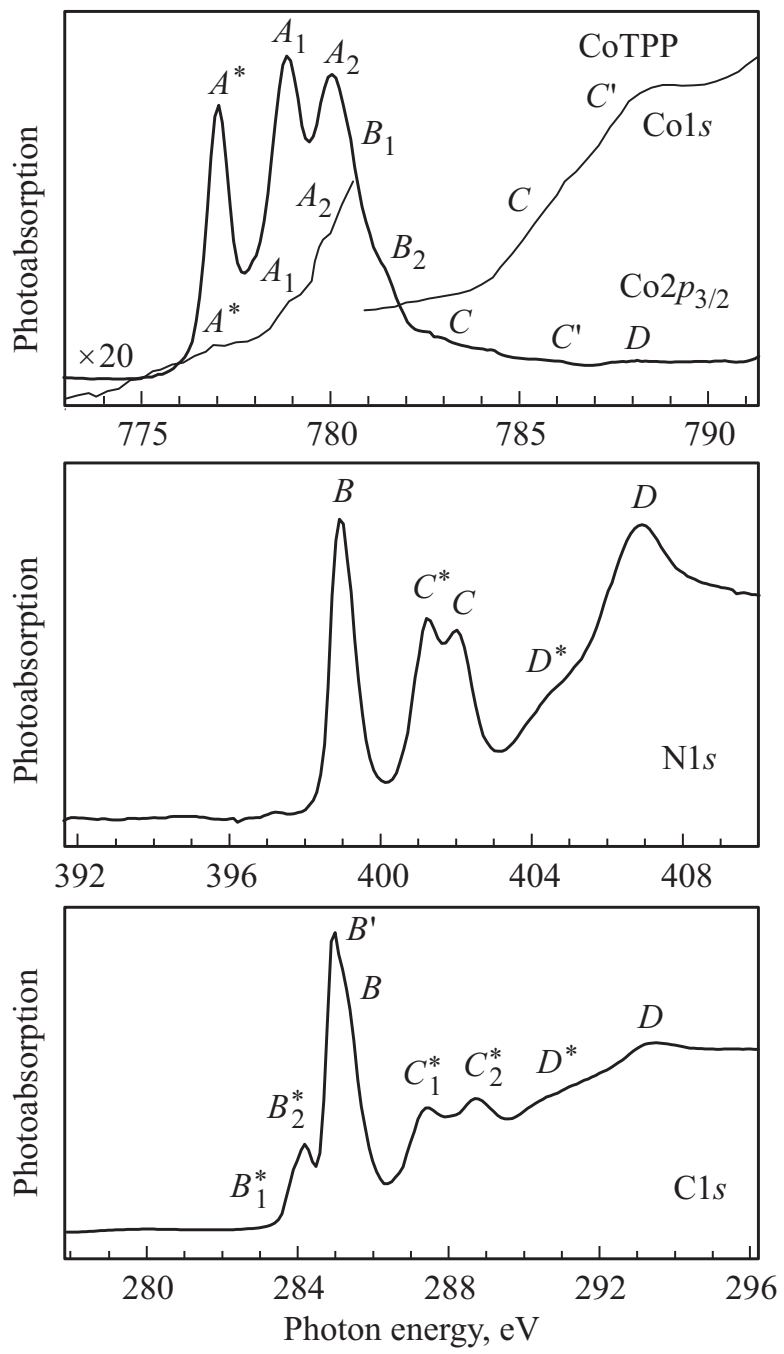

Рис. 5. Энергетически совмещенные Co2p $p_{3 / 2-}$, Co1s-, $\mathrm{N} 1 s$ - и $\mathrm{C} 1 s$-спектры поглощения тетрафенилпорфирина кобальта СоТРР. Энергетические шкалы совмещены с учетом разницы между энергиями связи остовных уровней $\Delta E\left(\mathrm{Co} 2 p_{3 / 2}-\mathrm{N} 1 s\right)=381.3 \mathrm{eV}$ и $\Delta E(\mathrm{~N} 1 s-\mathrm{C} 1 s)=113.8 \mathrm{eV}$. $\mathrm{Co} 1 s$-спектр приведен к шкале $\mathrm{Co} 2 p_{3 / 2}$-спектра, используя энергию характеристической $K \alpha_{1}\left(1 s \rightarrow 2 p_{3 / 2}\right)$-перехода в металлическом кобальте $(6930.32 \mathrm{eV}[47])$.

щения имеет аналог в форме малоинтенсивной полосы только в Ni1s-спектре, тогда как в $\mathrm{N} 1 s$ - и $\mathrm{C} 1 s$-спектрах соответствующие абсорбционные полосы отсутствуют. В работах $[27,28]$, где исследовались Ni2 $p_{3 / 2}$-NEXAFS спектры поглощения ряда порфиринов никеля, также отмечался подобный результат, который объяснялся тем, что $b_{1 g}$-МО является несвязывающей орбиталью, построенной в основном из атомных $\mathrm{Ni} 3 d_{x^{2}-y^{2}}$-состояний с очень малой примесью $\mathrm{N} 2 p$-состояний, в результате чего соответствующий переход $\mathrm{N} 1 s \rightarrow 3 d b_{1 g} \quad$ не наблюдается в спектре поглощения азота. Однако согласно DFT-расчетам электронной структуры NiTPP и других порфиринов никеля $[37,50]$, низшая свободная $b_{1 g}$-MO (lowest unoccupied MO, LUMO) помимо вклада

$\mathrm{Ni} 3 d_{x^{2}-y^{2}}$-состояний содержит также существенный вклад $(\sim 30 \%) 2 p$-состояний атомов азота, и, следовательно, этот вклад должен проявляться в $\mathrm{N} 1 s$-спектре поглощения в виде достаточно интенсивного низкоэнергетического пика. Его отсутствие удается понять, если учесть донорно-акцепторный механизм формирования химической $\sigma$-связи между атомом никеля и атомами азота. Согласно этому механизму каждая неподеленная $2 p$-электронная пара четырех атомов азота смещается в область свободного $\mathrm{Ni} 3 d_{x^{2}-y^{2}}$-состояния и формирует двухэлектронную ковалентную связь между атомом никеля и атомом азота, которая заметно отличается от обычной двуцентровой ковалентной связи [38]. Таким образом, наряду со свободными $\mathrm{Ni} 3 d$-состояниями в образовании LUMO участвуют также занятые $2 p$-состояния атомов азота, которые представляют собой неподеленные электронные пары. В этом случае переходы N1s-электронов в LUMO попросту невозможны, поскольку N2p-состояния в ней заняты. Это объяснение не противоречит проявлению $b_{1 g}$-MO в Ni1s-спектре NiTPP в форме квадрупольного перехода малой интенсивности. Заметим, что аналогичные соображения были использованы нами ранее при рассмотрении NEXAFSспектров простейшего порфирина никеля $\mathrm{NiP}$ [29].

Абсорбционный резонанс $B$, отчетливо наблюдаемый в Ni2 $2 p_{3 / 2^{-}}$и $\mathrm{N} 1 s$-спектрах NiTPP, в точности соответствует полосе $B$ в спектрах $\mathrm{NiP}$ (рис. 2 и 3). Как и в случае соответствующих спектров NiP [29], этот пик логично связать с переходами остовных электронов на $e_{g}$-МО $\pi$-симметрии, в образовании которой участвуют $\mathrm{Ni} 3 d_{x z, y z}, \mathrm{~N} 2 p-$ и $\mathrm{C}_{\alpha} 2 p-\mathrm{AO}$ атомов, входящих в состав квазимолекулы $\mathrm{MN}_{4}\left(\mathrm{C}_{\alpha}\right)_{8}$. Переходы $\mathrm{C}_{\alpha} 1 s$-электронов на $e_{g}$-МО, по-видимому, ответственны за слабое плечо $B$ в спектре поглощения углерода. Значительная интенсивность полосы в $\mathrm{Ni} 2 p_{3 / 2}$-спектре поглощения NiTPP означает, что в этом комплексе, как и в NiP, сохраняется заметная делокализация $3 d-$-электронной плотности. В работах [27-29] подобная делокализация в порфиринах и фталоцианине никеля была связана с сильным $\pi$-связыванием между атомами никеля и лиганда. Это связывание осуществляется за счет ковалентного $\mathrm{Ni} 3 d_{x z, y z}-\mathrm{N}, \mathrm{C} 2 p$ смешивания и сопровождается зарядовым переносом Ni3 $d$-электронной плотности на атомы лиганда (эффект обратного донирования [44]).

Между резонансами $A$ и $B$, однозначно идентифицируемыми в $\mathrm{Ni} 2 p_{3 / 2}$ - и $\mathrm{N} 1 s$-спектрах NiTPP, располагаются абсорбционные структуры $B_{1}^{*}, B_{2}^{*}$ и $B^{\prime}$, которые наблюдаются только в C1s-спектре NiTPP (рис. 4). Как следует из сравнения этого спектра с $\mathrm{C} 1 s$-спектром $\mathrm{NiP}$ и молекулы бифенила (рис. 3), эти структуры обусловлены рентгеновскими возбуждениями атомов углерода порфинового лиганда $\left(B_{1}^{*}, B_{2}^{*}\right)$ и фенильной группы $\left(B^{\prime}\right)$. Как было отмечено выше, плечо $B$ с высокоэнергетической стороны полосы $B^{\prime}$ связано, повидимому, с $1 s$-переходами в $\mathrm{C}_{\alpha}$-атомах в порфиновом лиганде. Таким образом, низкоэнергетические полосы $B_{1}^{*}$ и $B_{2}^{*}$ в $\mathrm{C} 1 s$-спектре поглощения порфинового лиганда 
в NiTPP (рис. 3) отражают изменения в химическом состоянии атомов углерода порфинового макроцикла и структуре его MO в результате присоединения к NiP четырех фенильных групп. Это приводит к появлению в NiTPP свободных электронных состояний в энергетическом интервале между $\sigma b_{1 g}(A)$ - и $\pi e_{g}(B)$-состояниями, образуемыми центральной областью $(\mathrm{NiP})$ в NiTPP.

Далее, возбужденное состояние, ответственное за абсорбционную полосу $C^{*}$, явно наблюдается, повидимому, только в $\mathrm{N} 1 s$-спектре и отсутствует в спектрах никеля и углерода. Это позволяет предполагать, что эта полоса связана с переходами $\mathrm{N} 1 s$-электронов на несвязывающую свободную МО $\pi$-типа $\left(b_{2 u}\right.$ или $\left.a_{2 g}\right)$ порфинового макроцикла. В работах [22,24] полоса $C^{*}$ из-за недостаточного разрешения наблюдалась в $\mathrm{N} 1 s$-спектрах ZnTPP и СоTРP вместе с полосой $C$ в виде одной полосы, которая на основании $\mathrm{CNDO} / \mathrm{S} 2$-расчетов была связаны с электронными переходами на две близко расположенные $\pi b_{2 u^{-}}$и $\pi e_{g}-\mathrm{MO}$, локализованные на пиррольных кольцах порфинового макроцикла. Отсутствие этой абсорбционной полосы в $\mathrm{C} 1 s$-спектре обусловлено, по-видимому, ее слабостью на фоне доминирующих абсорбционных структур фенильных групп.

Полосы поглощения $C_{1}^{*}$ и $C_{2}^{*}$ в $\mathrm{C} 1 s$-спектре NiTPP, как было ранее показано (рис. 3), связаны с возбуждением $1 s$-электронов атомов углерода фенильных групп в свободные электронные $\sigma^{*}(\mathrm{C}-\mathrm{H})$ - и $2 \pi^{*}$-состояния последних.

Наконец, рассмотрим происхождение полосы $C$, которая отчетливо наблюдается в $\mathrm{Ni} 1 s^{-}, \mathrm{Ni}_{2} p_{3 / 2^{-}}$и $\mathrm{N} 1 s$-спектрах поглощения (рис. 4). В $1 s$-спектрах поглощения $3 d$-атомов в плоских комплексах подобная полоса обычно связывается с переходами остовных электронов на $a_{2 u}$-МО $\pi$-типа с вкладом $4 p_{z}$-состояний атома металла [51], которая в нашем случае образована $4 p_{z}$-AO атома металла и $2 p$-состояниями атомов $\mathrm{N}$ и С. Такое строение этой МО объясняет ее проявление в $1 s$-спектрах поглощения атомов никеля и азота, поскольку на нее разрешены дипольные переходы Ni1s- и N1s-электронов. В C1s-спектре подобные переходы не видны вследствие доминирования $\mathrm{C} 1 s$-возбуждений фенильных групп. Однако такая идентификация полосы $C$ не объясняет ее заметную интенсивность в $\mathrm{Ni} 2 p_{3 / 2}$-спектре поглощения: переходы $\mathrm{Ni} 2 p_{3 / 2} \rightarrow 4 p_{z}$ дипольно запрещены. Поэтому логично считать, что в спектре свободных электронных состояний NiTPP вблизи $a_{2 u}$-MO располагается MO, переходы на которую дипольно разрешены для $\mathrm{Ni} 2 p_{3 / 2}$-электронов. Вполне вероятно, что таковой является разрыхляющая $a_{1 g}$-MO $\sigma$-типа, которая образована из $\mathrm{Ni} 4 s-, 3 d_{z^{2-}}$ и $\mathrm{N} 2 p$-состояний и поэтому дипольно доступна для абсорбционных переходов $1 s$-электронов атомов никеля и азота. Заметим, что мы не можем полностью исключить, что переходы $\mathrm{N} 1 s$ и $\mathrm{Ni} 2 p_{3 / 2}$-электронов ответственны также за появление плеча $D^{*}$ в соответствующих спектрах.

Рассмотрим теперь спектры СоТРР (рис. 5). Со2 $2 p_{3 / 2}$, $\mathrm{N} 1 s-$ и $\mathrm{C} 1 s$-спектры СоТРР представлены на сво- их энергетических шкалах, которые совмещены, используя разности энергий связи остовных уровней $\Delta E\left(\mathrm{Co} 2 p_{3 / 2}-\mathrm{N} 1 s\right)=381.3 \mathrm{eV}$ и $\Delta E(\mathrm{~N} 1 s-\mathrm{C} 1 s)=$ $=113.8 \mathrm{eV}$, измеренных методом фотоэлектронной спектроскопии. Заметим, что полученные величины всего в пределах точности эксперимента $(0.1-0.3 \mathrm{eV})$ совпадают с данными работ для СоТРР $[11,48]$. Как и в случае NiTPP, использование C $1 s$-спектра СоТРP при рассмотрении всего набора спектров ограничено, поскольку его структура отражает главным образом рентгеновские возбуждения фенильных атомов углерода, доминирующих в молекулярном комплексе. Энергетическое совмещение $\mathrm{Co} 1 s$-спектра поглощения с $\mathrm{Co} 2 p_{3 / 2}$-спектром выполнено, используя энергию рентгеновской характеристической $K \alpha_{1}\left(1 s \rightarrow 2 p_{3 / 2}\right)$-линии для металлического кобальта (6930.32 eV [47]).

При сравнении энергетически совмещенных $\mathrm{Co} 2 p_{3 / 2}$, $\mathrm{Co} 1 s$ - и $\mathrm{N} 1 s$-спектров поглощения СоТРР (рис. 5) видно, что низшее свободное состояние комплекса задает полоса поглощения $A^{*}$. Выше было показано, что она связана с переходами $2 p_{3 / 2-}$ и $1 s$-электронов атома кобальта на частично заполненную $3 d b_{2 g}$-МО $\pi$-типа. Затем следует свободная слабо разрыхляющая $3 d b_{1 g}$-MO $\sigma$-типа, электронные переходы на которую отображаются в спектрах кобальта в виде триплетного и синглетного компонентов $\left(A_{1}\right.$ и $\left.A_{2}\right)$ вследствие обменного $3 d-3 d$ взаимодействия электронов на частично заполненных $3 d b_{2 g}$ - и $3 d b_{1 g}$-МО. Несмотря на гибридизированный $\left(\mathrm{Co} 3 d_{x^{2}-y^{2}}+\mathrm{N} 2 p\right)$-характер $b_{1 g}$-MO в $\mathrm{N} 1 s$-спектре не наблюдаются абсорбционные полосы, соответствующие полосам $A_{1}$ и $A_{2}$. Как и в случае NiTPP и NiP, этот факт является следствием невозможности $\mathrm{N} 1 s \rightarrow b_{1 g}$-MO переходов, поскольку вклад в эту МО дают неподеленные пары N2p-электронов [29].

Следующее свободное состояние наблюдается как одиночная полоса $B$ в $\mathrm{N} 1 s$-спектре и двойная $B_{1}$ и $B_{2}$ в $2 p_{3 / 2}$-спектре кобальта. Представляется логичным приписать эти полосы, как и в случае спектров NiTPP и $\mathrm{NiP}$, переходам остовных электронов атомов азота и кобальта на $e_{g}$-МО $\pi$-типа с гибридизированным $\left(\mathrm{Co} 3 d_{x z, y z}+\mathrm{N} 2 p\right)$-характером, рассматривая дублетную структуру перехода как результат обменного $3 d-3 d$ электронного взаимодействия в конечном состоянии. Необходимо заметить, что взаимное энергетическое расстояние между полосами $A_{1}\left(A_{2}\right)$ и $B_{1}\left(B_{2}\right)$ в $2 p_{3 / 2}$-спектре кобальта СоТРР $(\sim 1.4 \mathrm{eV})$ заметно меньше расстояния между соответствующими полосами $A$ и $B$ в $2 p_{3 / 2}$-спектре никеля NiTPP $(1.9 \mathrm{eV})$. Это значительное уменьшение, очевидно, отражает изменения в спектре свободных электронных состояний комплексов МТРР, происходящие при замене атома никеля атомом кобальта.

В пользу заметной перестройки спектра свободных электронных состояний в СоTРP по сравнению с NiTPP говорит также тот факт, что низкоэнергетическая полоса $B_{1}^{*}-B_{2}^{*}$, располагающаяся в $\mathrm{C} 1 s$-спектре NiTPP между полосами $A$ и $B$ в $\mathrm{N} 1 s$-спектре (рис. 4 ), заметно сдвинута 
в спектре СоТРР (рис. 5) в сторону полосы $A_{1}\left(A_{2}\right)$. В результате свободное состояние комплекса СоТРР, ответственное за эту полосу, энергетически оказывается ниже $3 d b_{1 g}$-MO, с электронными переходами на которую связаны абсорбционные полосы $A_{1}$ и $A_{2}$ в $2 p_{3 / 2}$-спектре поглощения кобальта.

Заканчивая рассмотрение спектров поглощения СоТРР, заметим, что четкая полоса $C$, имеющая близкие энергетические положения в совмещенных спектрах никеля и азота NiTPP (рис. 4), в спектрах кобальта СоТРР состоит из двух слабо выраженных структур $C$ и $C^{\prime}$. При сравнении спектров кобальта с N1s-спектром можно отметить определенную корреляцию энергетического положения структуры $C-C^{\prime}$ в спектрах кобальта со структурами $C$ и $D^{*}$ в спектре азота. Это позволяет предполагать, что она, как и в случае NiTPP, связана с электронными переходами на МО, имеющими значительные вклады $\mathrm{Co} 4 p$-, $\mathrm{Co} 4 s$ - и $\mathrm{N} 2 p$-состояний.

\section{4. Заключение}

Методами рентгеновской абсорбционной спектроскопии изучены энергетические распределения и свойства нижних свободных электронных состояний в комплексах тетрафенилпорфиринов кобальта СоТРP и никеля NiTPP.

Анализ экспериментальных NEXAFS спектров, измеренных в области $2 p$ - и $1 s$-порогов ионизации комплексообразующих атомов металлов, а также $1 s$-порогов атомов лиганда (азота и углерода), выполнен на основе сравнения соответствующих спектров между собой и со спектрами простейшего порфирина никеля NiP. Установлено, что при общем подобии структуры спектров азота и углерода для СоТРP и NiTPP тонкая структура $2 p$ - и $1 s$-спектров поглощения атомов кобальта и никеля отличается радикальным образом друг от друга. Наблюдаемые отличия спектров кобальта и никеля связываются с особенностями энергетического распределения свободных электронных состояний с $3 d$-характером. Наличие в СоТРР частично заполненной валентной $3 d b_{2 g}$-МО обусловливает появление в спектрах кобальта низкоэнергетической полосы, отсутствующей в спектрах никеля NiTPP. Первые дипольно разрешенные переходы $2 p_{3 / 2}$-электронов никеля в NiTPP на $b_{1 g^{-}}$и $e_{g}$-MO в соответствующем спектре СоТРР обнаруживают дублетную структуру вследствие парамагнетизма последнего, поскольку в конечных состояниях этих переходов, $\left(3 d b_{2 g}^{1} 3 d b_{1 g}^{1}\right)$ и $\left(3 d b_{2 g}^{1} 3 d e_{g}^{1}\right)$, между $3 d$-электронами в частично заполненных $3 d b_{2 g}$ и $3 d b_{1 g}$ - или $3 d e_{g}-\mathrm{MO}$ возникает дополнительное (обменное) взаимодействие, которое расщепляет конечные состояния на два - синглетное $(S=0)$ и триплетное $(S=1)$, приводя к появлению в спектрах поглощения СоТРР для каждого из этих переходов двух абсорбционных резонансов вместо одного в спектрах NiTPP. Аналогичный эффект наблюдается и для квадрупольного перехода $1 s \rightarrow 3 d b_{1 g}$-МO в $1 s$-спектрах атомов металла при переходе от NiTPP к CoTPP.

Тонкая структура всех спектров поглощения NiTPP и СоТРР, энергетически совмещенных с учетом разности энергий связи остовных электронов атомов металла и лиганда, рассмотрена в рамках квазимолекулярного $\mathrm{MN}_{4}\left(\mathrm{C}_{\alpha}\right)_{8}$ подхода. Установлено, что спектр свободных состояний CoTPP отличается от спектра NiTPP за счет наличия низшей частично заполненной $3 d b_{2 g}$-MO и меньшего энергетического расстояния между $3 d b_{1 g}$-МO и $e_{g}$-MO. Отсутствие в $\mathrm{N} 1 s$-спектрах обоих комплексов переходов на $3 d b_{1 g}$-МО, имеющую гибридизированный $\mathrm{Ni}(\mathrm{Co}) 3 d-\mathrm{N} 2 p$ характер, связывается с донорноакцепторным характером химической связи между атомами металла и азота. В этом случае в образовании химической связи участвуют неподеленные пары $2 p$-электронов пары атомов азота, что приводит к невозможности переходов $\mathrm{N} 1 s$-электронов на $3 d b_{1 g}$-МO, поскольку $\mathrm{N} 2 p$-состояния в ней заняты. Спектр свободных электронных состояний СоТРР отличается от спектра NiTPP также другим положением несвязывающих МO с C2p-характером порфинового лиганда: в СоТРР эти MO энергетически находятся ниже $3 d b_{1 g}$-MO, тогда как в NiTPP они располагаются выше $3 d b_{1 g}$-MO.

Авторы выражают благодарность М.М. Бржезинской (БЭССИ), Е.М. Загребиной и А.В. Захаровой (СПбГУ) за помощь при проведении измерений.

\section{Список литературы}

[1] The Porphyrin Handbook / Ed. K.M. Kadish, K.M. Smith, R. Guilard. V. 1-10. Academic, San Diego, CA (2000).

[2] J.P. Collman, R. Boulatov, C.J. Sunderland, L. Fu. Chem. Rev. 104, 561 (2004).

[3] H.L. Anderson. Chem. Commun. 2323 (1999).

[4] O. Senge, M. Fazekas, E.G.A. Notaras, W.J. Blau, M. Zawadzka, O.B. Locos, E.M. Ni Mhuiercheartaigh. Adv. Mater. 19, 2737 (2007).

[5] P. Bhyrappa, J.K. Young, J.S. Moore, K.S. Suslick. J. Am. Chem. Soc. 118, 5708 (1996).

[6] M. Ethirajan, Y. Chen, P. Joshi, R.K. Pandey. Chem. Soc. Rev. 40, 340 (2011).

[7] D. Filippini, A. Alimelli, C. Di Natale, R. Paolesse, A. D’Amico, I. Lundström. Angew. Chem. Int. Ed. 45, 3800 (2006).

[8] A. Yella, H.-S. Lee, H.N. Tsao, C. Yi, A.K. Chandiran, Md.K. Nazeeruddin, E.W.-G. Diau, C.-Y. Yeh, S.M. Zakeeruddin, M. Grätzel. Science 334, 629 (2011).

[9] Z. Liu, A.A. Yasseri, J.S. Lindsey, D.F. Bocian. Science 302, 1543 (2003).

[10] W. Auwärter, K. Seufert, F. Klappenberger, J. Reichert, A. Weber-Bargioni, A. Verdini, D. Cvetko, M. Dell'Angela, L. Floreano, A. Cossaro, G. Bavdek, A. Morgante, A.P. Seitsonen, J.V. Barth. Phys. Rev. B 81, 245403 (2010).

[11] L. Scudeiro, D.E. Barlow, K.W. Hipps. J. Phys. Chem. B 104, 11899 (2000).

[12] L. Scudeiro, K.W. Hipps, D.E. Barlow. J. Phys. Chem. B 107, 2903 (2003). 
[13] L.G. Teugels, L.G. Avila-Bront, S.J. Sibener. J. Phys. Chem. C 115, 2826 (2011).

[14] S. Fatayer, R.G.A. Veiga, M.J. Prieto, E. Perim, R. Landers, R.H. Miwa, A. de Siervo. Phys. Chem. Chem. Phys. 17, 18344 (2015).

[15] T. Lukasczyk, K. Flechtner, L.R. Merte, N. Jux, F. Maier, J.M. Gottfried, H.-P. Steinrück. J. Phys. Chem. C 111, 3090 (2011).

[16] A. Weber-Bargioni, W. Auwärter, F. Klappenberger, J. Reichert, S. Lefrancois, T. Strunskus, C. Wölf, A. Schiffrin, Y. Pennec, J.V. Barth. Chem. Phys. Chem. 9, 89 (2008).

[17] Y. Bai, M. Sekita, M. Schmid, T. Bischof, H.-P. Steinrück, J.M. Gottfried. Phys. Chem. Chem. Phys. 12, 4336 (2010).

[18] J.M. Gottfried, K. Flechtner, A. Kretschmann, T. Lukasczyk, H.-P. Steinrück. J. Am. Chem. Soc. 128, 5644 (2006).

[19] M. Chen, X. Feng, L. Zhang, H. Ju, Q. Xu, J. Zhu, J.M. Gottfried, K. Ibrahim, H. Qian, J. Wang. J. Phys. Chem. C 114, 9908 (2010).

[20] K. Diller, F. Klappenberger, M. Marschall, K. Hermann, A. Nefedov, Ch. Wöll, J.V. Barth. J. Chem. Phys. 136, 014705 (2012).

[21] J. Stöhr. NEXAFS Spectroscopy. Springer Ser. Surf. Sci. Springer-Verlag, Berlin. (1992). V. 25. 403 p.

[22] S. Narioka, H. Ishii, Y. Ouchi, T. Yokayama, T. Ohta, K. Seki. J. Phys. Chem. 99, 1332 (1995).

[23] L.X. Chen, W.J.H. Jäger, G. Jennings, D.J. Gosztola, A. Munkholm, J.P. Hessler. Science 292, 262 (2001).

[24] T. Okajima, Y. Yamamoto, Y. Ouchi, K. Seki. J. Electron Spectrosc. Relat. Phenom. 114-116, 849 (2001).

[25] M.P. de Jong, R. Friedlein, S.L. Sorensen, G. Öhrwall, W. Osikowicz, C. Tengsted, S.K.M. Jönsson, M. Fahlman, W.R. Salaneck. Phys. Rev. B 72, 035448 (2005).

[26] N. Schmidt, R. Fink, W. Hieringer. J. Chem. Phys. 133, 054703 (2010).

[27] S.A. Krasnikov, A.B. Preobrajenski, N.N. Sergeeva, M.M. Brzhezinskaya, M.A. Nesterov, A.A. Cafolla, M.O. Senge, A.S. Vinogradov. Chem. Phys. 332, 318 (2007).

[28] S.A. Krasnikov, N.N. Sergeeva, M.M. Brzhezinskaya, A.B. Preobrajenski, Y.N. Sergeeva, N.A. Vinogradov, A.A. Cafolla, M.O. Senge, A.S. Vinogradov. J. Phys.: Condens. Matter 20, 235207 (2008).

[29] Г.И. Свирский, Н.Н. Сергеева, С.А. Красников, Н.А. Виноградов, Ю.Н. Сергеева, А.А. Cafolla, А.Б. Преображенский, А.С. Виноградов. ФТТ 59, 2, 357 (2017).

[30] K.A. Simonov, A.S. Vinogradov, M.M. Brzhezinskaya, A.B. Preobrajenski, A.V. Generalov, A.Yu. Klyushin. Appl. Surf. Sci. 267, 132 (2013)

[31] S.I. Fedoseenko, I.E. Iossifov, S.A. Gorovikov, J.-S. Schmid, R. Follath, S.L. Molodtsov, V.K. Adamchuk, G. Kaindl. Nucl. Insrum. Meth. Phys. Res. A 470, 84 (2001).

[32] http://www.sigmaaldrich.com

[33] А.П. Лукирский, И.А. Брытов. ФТТ 6, 43 (1964).

[34] W. Gudat, C. Kunz. Phys. Rev. Lett. 29, 169 (1972).

[35] A.S. Vinogradov, S.I. Fedoseenko, S.A. Krasnikov, A.B. Preobrajenski, V.N. Sivkov, D.V. Vyalikh, S.L. Molodtsov, V.K. Adamchuk, C. Laubschat, G. Kaindl. Phys. Rev. B 71, 045127 (2005).

[36] A.A. Chernyshov, A.A. Veligzhanin, Y.V. Zubavichus. Nucl. Insrum. Meth. Phys. Res. A 603, 95 (2009).

[37] M.-S. Liao, S. Scheiner. J. Chem. Phys. 117, 205 (2002).

[38] C. Berrios, G.I. Cárdenas-Jirón, J.F. Marco, C. Gutiérrez, M.S. Ureta-Zañartu. J. Phys. Chem. A 111, 2706 (2007).
[39] W.R. Scheidt, Y.J. Lee. Struct. Bonding 64, 1 (1987).

[40] H. Ebert, J. Stöhr, S.S.P. Parkin, M. Samant, A. Nilsson. Phys. Rev. B 53, 16067 (1996).

[41] J.G. Chen. Surf. Sci. Rep. 30, 1(1997).

[42] J. Wang, G. Cooper, D. Tulumello, A.P. Hitchcock. J. Phys. Chem. A 109, 10886 (2005).

[43] G.L. Miessler, P.J. Fischer, D.A. Tarr. Inorganic chemistry. Fifth edition. Upper Saddle River, New Jersey, Pearson (2014).

[44] И.Б. Берсукер. Электронное строение и свойства координационных соединений. Введение в теорию. 3-е изд., перераб. Химия, Л. (1986). 288 с.

[45] А.С. Виноградов, Т.М. Зимкина, В.Н. Акимов, Б. Шларбаум. Изв. АН СССР. Сер. физ. 38, 3, 508 (1974).

[46] G.R. Wight, C.E. Brion. J. Electron Spectrosc. Rel. Phenom. 4, 313 (1974).

[47] J.A. Bearden. Rev. Mod. Phys. 39, 78 (1967).

[48] D.H. Karweik, N. Winograd. Inorg. Chem. 15, 10, 2336 (1976).

[49] T. Konishi, J. Kawai, M. Fujiwara, T. Kurisaki, H. Wakita, Y. Gohshi. X-Ray Spectrometry 28, 470 (1999).

[50] A. Rosa, G. Ricciardi, E.J. Baerends, S.J.A. van Gisbergen. J. Phys. Chem. A 105, 3311 (2001).

[51] T.A. Smith, J.E. Penner-Hahn, M.A. Berding, S. Doniach, K.O. Hodgson. J. Am. Chem. Soc. 107, 5945 (1985). 